\title{
Simultaneous INTEGRAL and RXTE observations of the accreting millisecond pulsar HETE J1900.1-2455
}

\author{
M. Falanga ${ }^{1,2}$, J. Poutanen ${ }^{3}$, E. W. Bonning ${ }^{4}$, L. Kuiper ${ }^{5}$, J. M. Bonnet-Bidaud ${ }^{1}$, \\ A. Goldwurm ${ }^{1,2}$, W. Hermsen ${ }^{5,6}$, and L. Stella ${ }^{7}$ \\ ${ }^{1}$ CEA Saclay, DSM/DAPNIA/Service d'Astrophysique (CNRS FRE 2591), 91191 Gif-sur-Yvette, France \\ e-mail: mfalanga@cea.fr \\ 2 Unité mixte de recherche Astroparticule et Cosmologie, 11 place Berthelot, 75005 Paris, France \\ Astronomy Division, PO Box 3000, 90014 University of Oulu, Finland \\ ${ }^{4}$ Laboratoire de l'Univers et de ses Théories, Observatoire de Paris, 92195 Meudon Cedex, France \\ 5 SRON Netherlands Institute for Space Research, Sorbonnelaan 2, 3584 CA Utrecht, The Netherlands \\ 6 Astronomical Institute "Anton Pannekoek", University of Amsterdam, Kruislaan 403, 1098 SJ Amsterdam, The Netherlands \\ INAF-Osservatorio Astronomico di Roma, via Frascati 33, 00040 Monteporzio Catone (Roma), Italy
}

Received 27 September 2006 / Accepted 2 January 2007

\section{ABSTRACT}

\begin{abstract}
Aims. HETE J1900.1-2455 is the seventh known X-ray transient accreting millisecond pulsar and has been in outburst for more than one year. We compared the data on HETE J1900.1-2455 with other similar objects and made an attempt at deriving constraints on the physical processes responsible for a spectral formation.

Methods. The broad-band spectrum of the persistent emission in the $2-300 \mathrm{keV}$ energy band and the timing properties were studied using simultaneous INTEGRAL and publicly available RXTE data obtained in October 2005. The properties of the X-ray bursts observed from HETE J1900.1-2455 were also investigated.

Results. The spectrum is well described by a two-component model consisting of a blackbody-like soft X-ray emission at $0.8 \mathrm{keV}$ temperature and a thermal Comptonized spectrum with electron temperature of $30 \mathrm{keV}$ and Thomson optical depth $\tau_{\mathrm{T}} \sim 2$ for the slab geometry. The source is detected by INTEGRAL up to $200 \mathrm{keV}$ at a luminosity of $5 \times 10^{36} \mathrm{erg} \mathrm{s}^{-1}$ (assuming a distance of $5 \mathrm{kpc}$ ) in the 0.1-200 keV energy band. We have also detected one type I X-ray burst which shows photospheric radius expansion. The burst occurred at an inferred persistent emission level of $\sim 3-4 \%$ of the Eddington luminosity. Using data for all X-ray bursts observed to date from HETE J1900.1-2455, the burst recurrence time is estimated to be about 2 days. No pulsations have been detected either in the RXTE or in the INTEGRAL data which puts interesting constraints on theories of magnetic field evolution in neutron star low-mass $\mathrm{X}$-ray binaries.
\end{abstract}

Key words. stars: pulsars: individual: HETE J1900.1-2455 - stars: neutron - X-rays: binaries - X-rays: bursts

\section{Introduction}

The detection of X-ray millisecond pulsation in persistent emission from low-mass X-ray binaries (LMXBs) remained elusive for many years until the discovery of the first accreting millisecond pulsar (MSP) by Wijnands \& van der Klis (1998). Since that time, a total of seven accreting MSP transients have been detected. They are weakly magnetized $\left(\sim 10^{8}-10^{9} \mathrm{G}\right)$ neutron stars (NS) with spin frequencies in the $180-600 \mathrm{~Hz}$ range and orbital periods between $40 \mathrm{~min}$ and $5 \mathrm{~h}$ (see reviews by Wijnands 2006; Poutanen 2006). Their companion stars have been found to be either highly evolved white or brown dwarfs. For the first time, the predicted decrease of the NS spin period during accretion was measured in the accreting MSP IGR J00291+5934 (Falanga et al. 2005b). This provided a strong confirmation of the theory of "recycled" pulsars in which old neutron stars in LMXBs become millisecond radio pulsars through spin-up by transfer of angular momentum by the accreting material.

MSP energy spectra can be well described by a twocomponent model consisting of a soft black body (or multi-color blackbody) and a hard power-law like tail. The soft thermal component could be associated with radiation from the accretion disc and/or the heated NS surface around the shock (see e.g.
Gierliński \& Poutanen 2005; Poutanen 2006). The hard emission is likely to be produced by thermal Comptonization in the hot accretion shock on the NS surface (Gierliński et al. 2002; Poutanen \& Gierliński 2003) with seed photons coming from the stellar surface. The observed hard spectra are similar to the spectra observed from atoll sources in their hard, low-luminosity state (Barret et al. 2000).

HETE J1900.1-2455 was discovered during a bright X-ray burst by the High Energy Transient Explorer 2 (HETE-2) on 14 June 2005 (Vanderspek et al. 2005). Followup observations with the Rossi X-ray Timing Explorer (RXTE) identified the source as the seventh X-ray accreting millisecond pulsar, with a pulse frequency of $377.3 \mathrm{~Hz}$, an orbital period of $83 \mathrm{~min}$, and most likely a $0.016-0.07 M_{\odot}$ brown dwarf companion (Kaaret et al. 2006). The detected burst was consistent with a type I $\mathrm{X}$-ray burst with photospheric radius expansion. Assuming that the bolometric burst peak luminosity during photospheric radius expansion saturated at the Eddington limit, Kawai \& Suzuki (2005) estimated the distance to the source to be $\sim 5 \mathrm{kpc}$ assuming helium burst burning and canonical NS values.

An optical counterpart candidate had an $R$-band magnitude of 18.02 and a broad HeII emission line spectrum (Fox 2005; 


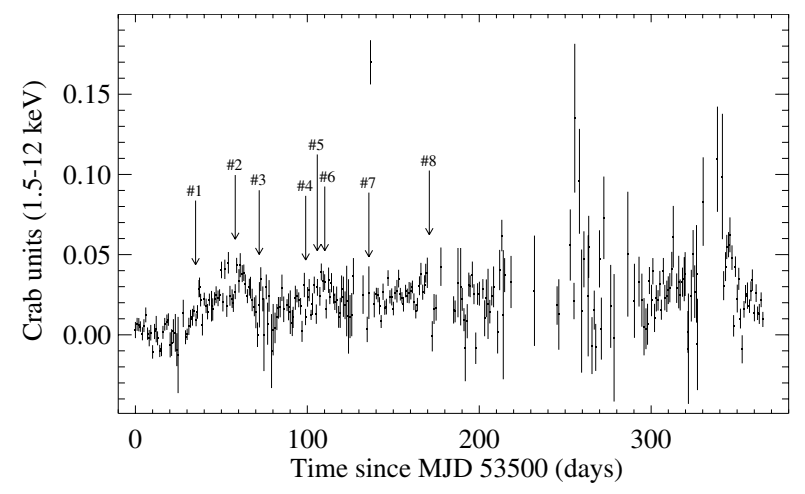

Fig. 1. RXTE/ASM light curve for HETE J1900.1-2455 averaged over 1-day intervals from May 5, 2005 (53500 MJD). The count rate has been converted into flux using 1 Crab Unit for $75 \mathrm{cts} \mathrm{s}^{-1}$ (Levine et al. 1996). The arrows indicate the times of the detected X-ray bursts (Vanderspek et al. 2005; Barbier et al. 2005; Galloway et al. 2005; and this work).

Steeghs et al. 2005a). A similar line was previously observed in IGR J00291+5934 (Roelofs et al. 2004; Filippenko et al. 2004). The optical counterpart of the X-ray source is located at coordinates $\alpha_{\mathrm{J} 2000}=19^{\mathrm{h}} 00^{\mathrm{m}} 08^{\mathrm{s}} .65$ and $\delta_{\mathrm{J} 2000}=-24^{\circ} 55^{\prime} 13^{\prime \prime} .7$ with an uncertainty of 0.2 . Near-infrared observations detected the optical candidate at a constant magnitude of $J=17.6$. No radio counterpart consistent with the HETE J1900.1-2455 coordinates was detected by the VLA (Steeghs et al. 2005b; Rupen et al. 2005).

One year after the discovery, HETE J1900.1-2455 is still active (see Fig. 1). Compared to other accreting MSPs with outburst periods of a few days to a month, HETE J1900.1-2455 shows evidence of being a "quasi-persistent" X-ray source. This source also has other properties atypical for accreting MSPs. During the first $\sim 30$ days after the discovery, HETE J1900.12455 showed significant flux emission variability in the fractional rms amplitude (Galloway et al. 2006). On July 8, 2005 (MJD 53559) during the flux brightening, the observed pulse frequency decreased by $\Delta v / v \sim 6 \times 10^{-7}$, and in the subsequent observations, the pulsations were suppressed (Kaaret et al. 2006).

In this paper we report the INTEGRAL observations of HETE J1900.1-2455 obtained simultaneously with RXTE. We study the broad-band spectral and timing properties of the source. The X-ray burst properties are also investigated.

\section{Observations and data}

\subsection{INTEGRAL}

The present data were obtained during the INTEGRAL (Winkler et al. 2003) Target of Opportunity (ToO) observation during satellite revolution 371, starting on October 27 and ending on October 29, 2005, with a total exposure time of $210 \mathrm{ks}$. The observation, aimed at HETE J1900.1-2455, consists of 64 stable pointings with a source position offset $<2$. 1 . We analyzed data from the IBIS/ISGRI coded mask telescope (Ubertini et al. 2003; Lebrun et al. 2003) at energies between 18 and $300 \mathrm{keV}$ and from the JEM-X monitor, module 1 (Lund et al. 2003) between 3 and $20 \mathrm{keV}$. The data reduction was performed using the standard Offline Science Analysis (OSA) version 5.1 distributed by the INTEGRAL Science Data Center (Courvoisier et al. 2003). The algorithms used for the spatial and spectral analysis are described in Goldwurm et al. (2003). The ISGRI light curves are based on events selected according to the detector illumination pattern for HETE J1900.1-2455. For ISGRI we used an illumination factor threshold of 0.25 for the energy range 18$40 \mathrm{keV}$; for JEM-X we used the event list of the whole detector in the 3-6 keV, 6-12 keV and 12-20 keV energy band.

We first deconvolved and analyzed separately the 64 single pointings and then combined them into a total mosaic image in the $20-40 \mathrm{keV}$ and 40-200 keV energy band, respectively. In the mosaic, HETE J1900.1-2455 is clearly detected at a significance level of $\sim 100 \sigma(20-40 \mathrm{keV})$ and $\sim 50 \sigma$ at higher energy (40-200 keV). The source position in the $20-40 \mathrm{keV}$ band is $\alpha_{\mathrm{J} 2000}=19^{\mathrm{h}} 00^{\mathrm{m}} 09^{\mathrm{s}} .19$ and $\delta_{\mathrm{J} 2000}=-24^{\circ} 55^{\prime} 12^{\prime \prime} .1$ (error of 0.4 at the 90 per cent confidence level, Gros et al. 2003), which is offset with respect to the optical position by 0'.13 (Fox 2005). The background-subtracted 20-40 and 40-200 keV light curves were extracted from the images using all available pointings, each with $\sim 3.3 \mathrm{ks}$ exposure. The source mean count rate was almost constant at $\sim 4.0 \mathrm{cts} \mathrm{s}^{-1}\left(\sim 3.0 \times 10^{-10} \mathrm{erg} \mathrm{cm}^{-2} \mathrm{~s}^{-1}\right)$ in the $20-40 \mathrm{keV}$ band and $\sim 2.8 \mathrm{cts} \mathrm{s}^{-1}\left(\sim 3.8 \times 10^{-10} \mathrm{erg} \mathrm{cm}^{-2} \mathrm{~s}^{-1}\right)$ in the $40-200 \mathrm{keV}$ energy band. The count rates were converted to un-absorbed flux using the Comptonization model described in Sect. 3.1.1.

\subsection{RXTE}

We used two RXTE ToO observations performed simultaneously with INTEGRAL between October 28 and 29, 2005 (observation id. 91432), for which the data were publicly available. The total net exposure times were 6.2 and $3.2 \mathrm{ks}$, respectively. We analyzed data from the Proportional Counter Array (PCA; 2-60 keV) (Jahoda et al. 1996) and the High Energy X-ray Timing Experiment (HEXTE; 15-250 keV) (Rothschild et al. 1998 ) on board the RXTE satellite. For the spectral analysis, we extracted the PCA (modules 0,2 and 3) energy spectrum using the standard software package FTOOLS version 6.0.2. For HEXTE, we used the ON-source data, using default screening criteria for Cluster 0. For the timing analysis we used all PCU data, which were on during the entire observation. The PCA data were collected in the E_125us_64M_0_1s event mode, recording event arrival times with $125 \mu$ s time resolution, and sorting events in 64 PHA channels. Default selection criteria were applied.

\subsection{Spectral analysis}

Broad-band spectral analysis was done using XSPEC version 11.3 (Arnaud 1996), combining the 3-22 keV RXTE/PCA data with the 3-22 keV INTEGRAL/JEM-X and 16-90 keV RXTE/HEXTE data with 20-300 keV INTEGRAL/ISGRI data. A multiplicative factor for each instrument was included in the fit to take into account the uncertainty in the cross-calibration of the instruments. The factor was fixed at 1 for the PCA data. A systematic error of $1 \%$ was applied to PCA/HEXTE and $2 \%$ to JEM-X/ISGRI spectra, which corresponds to the current uncertainties in the response matrix. All uncertainties in the spectral parameters are given at a $90 \%$ confidence level for single parameters. We use a source distance of $5 \mathrm{kpc}$ throughout the paper.

\section{Results}

\subsection{Persistent emission}

\subsubsection{Spectral properties}

We analyzed the JEM-X/ISGRI spectra independently both before and after the X-ray burst, and no significant spectral 
Table 1. Best-fit spectral parameters with GAUSSIAN + COMPPS (or cutoff PL) + BB (or DISKBB) model.

\begin{tabular}{|c|c|c|c|c|}
\hline & cutoff PL + BB & DISKBB & BB & BB \\
\hline$N_{\mathrm{H}}\left(10^{22} \mathrm{~cm}^{-2}\right)$ & 0.16 (f) & $0.16(f)$ & 0.16 (f) & 0.16 (f) \\
\hline$k T_{\text {in }}$ or $k T_{\mathrm{bb}}(\mathrm{keV})$ & $0.90_{-0.03}^{+0.03}$ & $1.0_{-0.03}^{+0.05}$ & $0.8_{-0.02}^{+0.02}$ & $0.7_{-0.01}^{+0.01}$ \\
\hline$R_{\mathrm{in}} \sqrt{\cos i}^{a}(\mathrm{~km})$ & - & $2.6_{-0.3}^{+0.2}$ & - & - \\
\hline$R_{\mathrm{bb}}^{a}(\mathrm{~km})$ & $5.0_{-06}^{+0.7}$ & -0.3 & $4.8_{-0 .}^{+0.7}$ & $4.4_{-0.5}^{+0.6}$ \\
\hline$E_{\mathrm{Fe}}(\mathrm{keV})$ & $6.41_{-0.14}^{+0.13}$ & $6.41_{-0.12}^{+0.14}$ & $6.42_{-0.15}^{+0.6 .1}$ & $6.41_{-0.14}^{+0.5 .12}$ \\
\hline$\sigma_{\mathrm{Fe}}(\mathrm{keV})$ & 0.5 (f) & 0.5 (f) & $0.5(\mathrm{f})$ & 0.5 (f) \\
\hline$k T_{\mathrm{e}}(\mathrm{keV})$ & - & $27.2_{-20}^{+1.2}$ & $27.9_{-1.4}^{+1.8}$ & $25.9_{-16}^{+1.9}$ \\
\hline$k T_{\text {seed }}(\mathrm{keV})$ & - & $1.4_{-0.32}^{+0.10}$ & 1.4 (f) & $=k T_{\mathrm{BB}}^{-1.6}$ \\
\hline$\tau_{\mathrm{T}}$ & - & $2.1^{+0.1}+x^{+0.1}$ & $2.0^{+0.06}$ & $2.2^{+0.1}$ \\
\hline$A_{\text {seed }}^{a}\left(\mathrm{~km}^{2}\right)$ & - & $13.8_{-0.04}^{+0.03}$ & $14.2_{-03}^{+0.3}$ & $240_{-29}^{+01}$ \\
\hline $\cos \theta$ & - & $0.59_{-0.07}^{+0.05}$ & $0.6(\mathrm{f})$ & 0.6 (f) \\
\hline$\Gamma$ & $1.60_{-0.03}^{+0.03}$ & $-\quad-0.01$ & - & - \\
\hline$E_{\text {cut }}(\mathrm{keV})$ & $59^{+3}-0.03$ & - & - & - \\
\hline$\chi^{2} /$ d.o.f. & $105^{-2} / 105$ & $114 / 103$ & $110 / 105$ & $117 / 106$ \\
\hline$L_{\mathrm{bu}}^{a}\left(10^{36} \mathrm{erg} \mathrm{s}^{-1}\right)$ & $5.0 \pm 0.5$ & $5.6 \pm 0.5$ & $4.9 \pm 0.5$ & $4.9 \pm 0.5$ \\
\hline
\end{tabular}

${ }^{a}$ Assuming a distance of $5 \mathrm{kpc}$.

variation was found. The burst occurred around the middle of the INTEGRAL observation. The RXTE observations were also performed before and after the X-ray burst, and the PCA/HEXTE spectral parameters were consistent with those determined from the INTEGRAL spectra. Therefore, we studied in detail the broad-band 3-300 keV spectrum of HETE J1900.1-2455 using the joint INTEGRAL and RXTE data. For the INTEGRAL data we removed the time interval corresponding to the burst. The energy range covered by JEM-X/PCA does not allow us to constrain the interstellar hydrogen column density, $N_{\mathrm{H}}$, well. Therefore, in all our spectral fits we fixed $N_{\mathrm{H}}$ at $1.6 \times 10^{21} \mathrm{~cm}^{-2}$, the value found from Swift observations at lower energies (Campana et al. 2005). This value is close to the Galactic value reported in the radio maps of Dickey \& Lockman (1990).

We first fit the joint JEM-X/ISGRI/PCA/HEXTE (3-300 keV) spectrum using a simple photoelectricallyabsorbed power-law, PL, model which was found inadequate with a $\chi^{2} /$ d.o.f. $=662 / 110$. A better fit was found by adding a blackbody, BB, model for the soft thermal emission and by replacing the PL with a cutoff PL model. This gave a $\chi^{2} /$ d.o.f. $=131 / 107$. The best-fit parameters are: blackbody temperature $k T_{\mathrm{BB}}=0.90 \pm 0.03 \mathrm{keV}$, a power-law photon index $\Gamma \sim 1.60 \pm 0.03$, and the cutoff energy $\sim 59_{-2}^{+3} \mathrm{keV}$. The PCA data show residuals in the $6-10 \mathrm{keV}$ range that can be fit by a broad Gaussian emission line. Correspondingly, the fit improved with a $\chi^{2} /$ d.o.f. $=105 / 105$. Confining the centroid of the line in the 6.3-6.8 keV range gave a line width of $\sigma=0.44 \pm 0.3$ (equivalent width $\simeq 102 \mathrm{eV}$ ); fixing the line width at $0.5 \mathrm{keV}$ constrains its position at $\simeq 6.41 \mathrm{keV}$.

In order to compare the HETE J1900.1-2455 spectrum with previously observed spectra of the same source class (Gierliński et al. 2002; Gierliński \& Poutanen 2005; Falanga et al. 2005a,b), we replaced the simple cutoff PL model with the thermal Comptonization model coMPPS in the slab geometry (Poutanen $\&$ Svensson 1996). The main model parameters are the Thomson optical depth $\tau_{\mathrm{T}}$ across the slab, the electron temperature $k T_{\mathrm{e}}$, the soft seed photon temperature $k T_{\text {seed }}$, and the inclination angle $\theta$ between the slab normal and the line of sight. The seed photons are assumed to be injected from the bottom of the slab. The soft thermal emission is fit by a simple blackbody BB or a multi-temperature disc blackbody DISKBB model (Mitsuda et al. 1984). The best fit parameters for the different models are

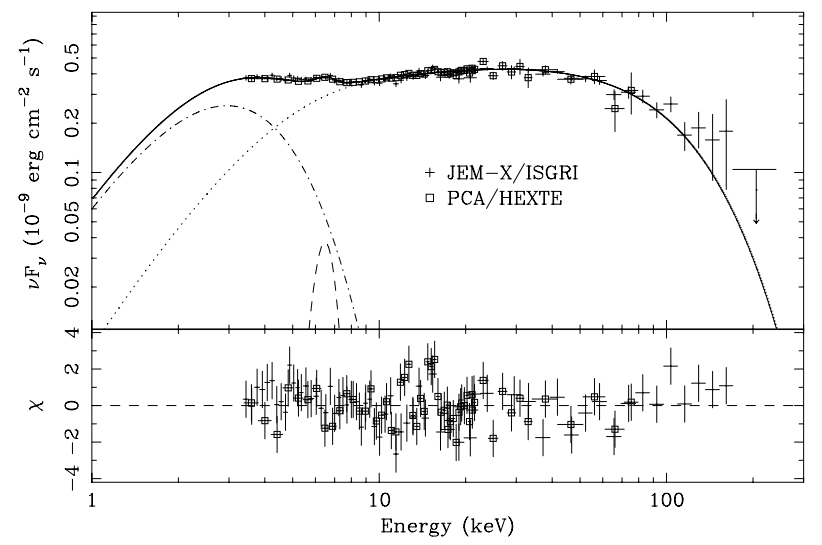

Fig. 2. The unfolded spectrum of HETE J1900.1-2455 fit with a COMPPS model plus a BB and a Gaussian line. The data points correspond to the PCA (3-22 keV), JEM-X (3-22 keV), HEXTE $(16-90 \mathrm{keV})$ and the ISGRI $(20-300 \mathrm{keV})$ spectra, respectively. The blackbody model is shown by a dot-dashed curve, the dotted curve gives the COMPPS model, the dashed curve is the Gaussian line, and the total spectrum is shown by the solid curve. The lower panel shows the residuals between the data and the model.

reported in Table 1. In Fig. 2, we show the unfolded spectrum and the residuals of the data to the BB plus COMPPS model.

The temperature of the soft thermal emission was found to be a factor $\sim 2$ higher than in other accreting millisecond pulsars (e.g., Gierliński \& Poutanen 2005; Falanga et al. 2005a). Since its discovery, this source has shown a rather high blackbody temperature (Kaaret et al. 2006). It was suggested for the millisecond pulsar XTE J1751-305 that the observed soft component could also be the source of the seed photons (Gierliński \& Poutanen 2005), therefore, we repeated the fit with $k T_{\text {soft }}=k T_{\text {seed }}$ and obtained only a marginally worse $\chi^{2} /$ d.o.f. $=117 / 106$. As discussed by Gierliński \& Poutanen (2005) and Kaaret et al. (2006), the true spectrum may consist of two black-bodies (disk and the heated surface of the NS). In adding another black-body component, we found that the new model does not improve the fit much. As the PCA/JEM-X energy range begins at $3 \mathrm{keV}$, it is impossible to search for additional soft emission in these data.

\subsubsection{Timing analysis}

We carried out a $Z_{1}^{2}$-statistic (Buccheri et al. 1983) period search of HETE J1900.1-2455 using barycentered (JPL DE200 solar system ephemeris) and orbital motion (Kaaret et al. 2006) corrected arrival times registered by the PCA instrument during the two short pointings of the ToO observation in the $2-10 \mathrm{keV}$ and 10-20 keV energy bands. We centered our periodicity search around the last measured frequency value $377.2959 \mathrm{~Hz}^{1}$, i.e. after the frequency shift observed on 53559 MJD (see Kaaret et al. 2006), with a rather wide frequency range of $\pm 0.007 \mathrm{~Hz}$. Within the scanned frequency range we did not find any indication of significant pulsations in either of the two energy bands. Thus, unless there was another frequency shift between 53567 and 53670 MJD such that the frequency range of our search was too small, there is no evidence for a pulsed signal from HETE J1900.1-2455 in the two short RXTE observations falling within the INTEGRAL observation.

${ }^{1}$ Note the frequency quoted in Kaaret et al. (2006), $377.291596(16) \mathrm{Hz}$ is a typo, the correct value is $377.29596(16) \mathrm{Hz}$. 


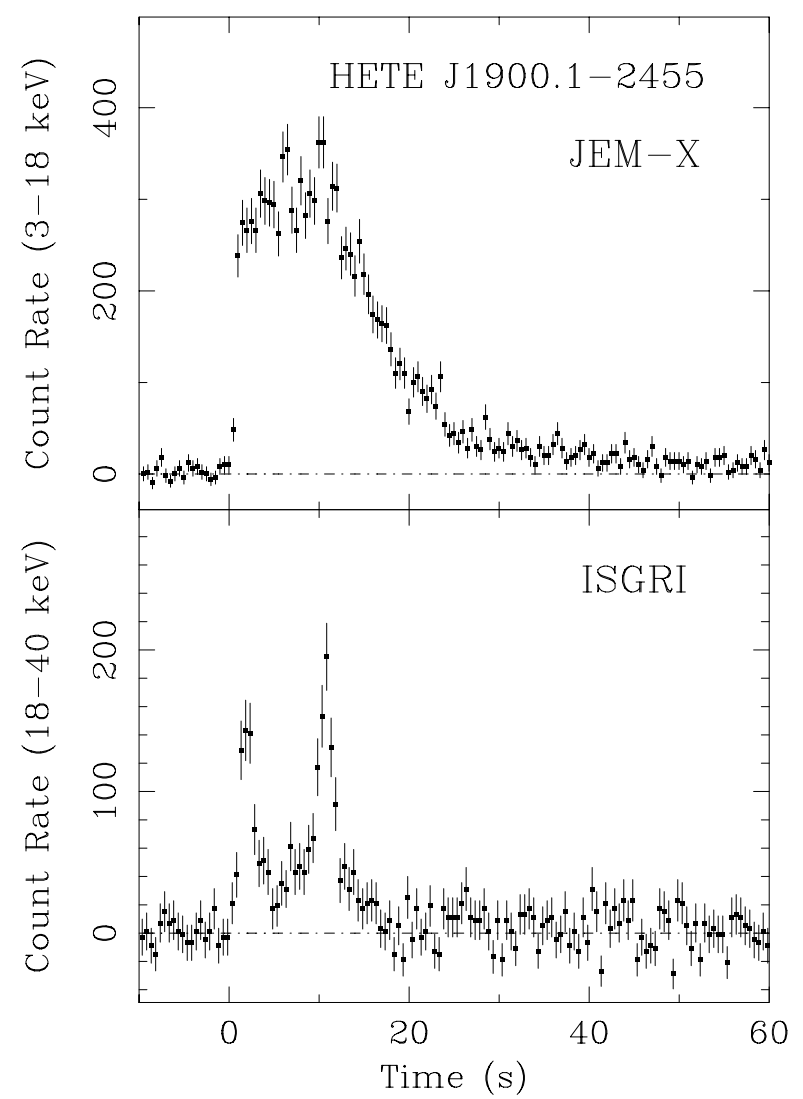

Fig. 3. A bright X-ray burst detected from HETE J1900.1-2455. The JEM-X (3-20 keV, upper panel) and IBIS/ISGRI (18-40 keV; lower panel) net light curves are shown (background subtracted). The time bin is $0.5 \mathrm{~s}$ for both IBIS/ISGRI and JEM-X light curve. At high energy the burst shows strong evidence of photospheric radius expansion.

Thinking that the pulsed fraction might increase above 20-30 keV, as was found for IGR J00291-5934 (Falanga et al. 2005b), we also searched the high-energy INTEGRAL data for the $2.65 \mathrm{~ms}$ period. No coherent pulsed signal was found in the $18-150 \mathrm{keV}$ IBIS/ISGRI band near the expected pulse frequency of HETE J1900.1-2455, confirming the suppression of the pulsed signal. Galloway et al. (2006) studied the pulsed fraction in detail and find an upper limit of about $1 \%$ rms during our observation.

\subsection{Properties of the $X$-ray bursts}

\subsubsection{Burst light curves}

In Fig. 3 we show the JEM-X and ISGRI burst light curve (28 October 2005, 10:25:12 UTC) in different energy bands. The burst rise time was $0.23 \pm 0.05 \mathrm{~s}$. The double peak profile is clearly evident at high energy (lower panel) within the first $12 \mathrm{~s}$, while during this time the intensity at lower energy (upper panel) remains constant. This can be interpreted as a consequence of a photospheric radius expansion (PRE) episode during the first part of the outburst (see e.g., Verbunt \& van den Heuvel 1995). When a burst undergoes a PRE episode, the luminosity remains nearly constant at the Eddington value, the atmosphere expands, and its temperature decreases, resulting in a double-peak profile observed at high energies. The tail of the burst at high energy can be seen for about $5 \mathrm{~s}$ after the PRE episode.

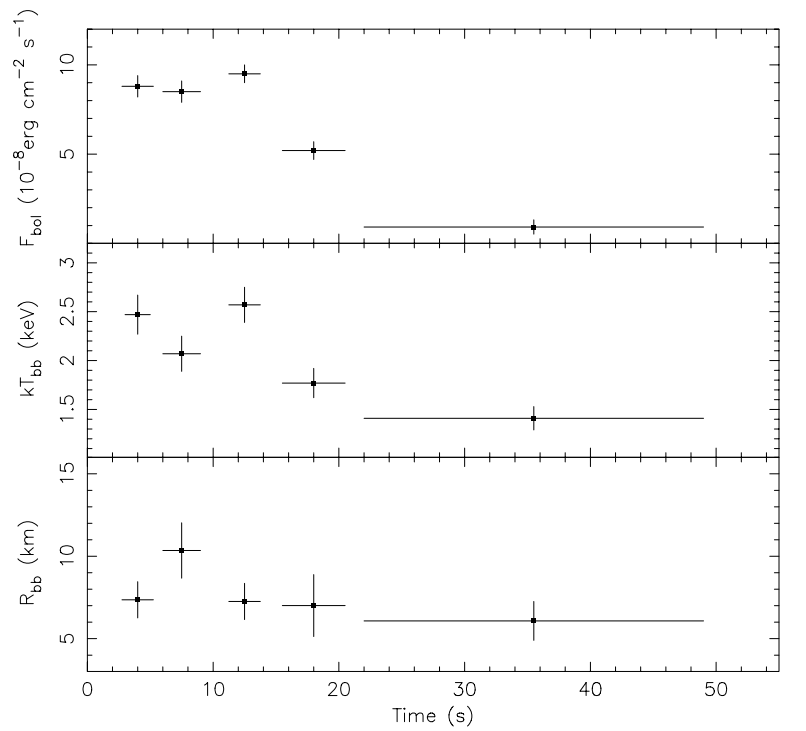

Fig. 4. Result of the X-ray burst time-resolved spectral analysis. The apparent blackbody radius was estimated assuming a source distance of $5 \mathrm{kpc}$.

\subsubsection{X-ray burst spectra}

We performed time-resolved analysis of the burst spectrum based on the JEM-X/ISGRI 3-50 keV energy band data. We verified that during the burst pointing the count rate was stable; we then used the JEM-X/ISGRI persistent emission spectrum as background. The burst is divided into time intervals as shown in Fig. 4, the shortest intervals being $\sim 3 \mathrm{~s}$ during the two spikes observed at high energy (see Fig. 4). The burst net spectrum was well fit by a photoelectrically absorbed blackbody $\left(\chi_{\text {red }}^{2} \sim 1.1\right)$. The inferred blackbody temperature, $k T_{\mathrm{bb}}$, and apparent blackbody radius, $R_{\mathrm{bb}}$, are shown in the middle and lower panels, respectively. During the first $12 \mathrm{~s}$, the un-absorbed bolometric flux was almost constant at $F_{\text {peak }}=9.5(2) \times 10^{-8} \mathrm{erg} \mathrm{cm}^{-2} \mathrm{~s}^{-1}$, while the blackbody temperature dropped in the middle, simultaneous with an increase by a factor of $\sim 1.5$ in blackbody radius. The observed temperature reached a peak at $\sim 2.5 \mathrm{keV}$, and then gradually decreased. The softening of the emission towards the end of the decay phase is also indicated by the e-folding decay times of $12.5 \pm 0.5 \mathrm{~s}$ in the $3-6 \mathrm{keV}$ to $4.3 \pm 0.7 \mathrm{~s}$ in the $12-20 \mathrm{keV}$ energy band. This behavior is typically observed during PRE X-ray bursts (e.g., Kuulkers et al. 2003).

The burst fluence, $f_{\mathrm{b}}=1.67(6) \times 10^{-8} \mathrm{erg} \mathrm{cm}^{-2}$, is calculated by integrating the measured $F_{\text {bol,bb }}$ over the burst duration of $\sim 50 \mathrm{~s}$. The effective burst duration is $\tau=f_{\mathrm{b}} / F_{\text {peak }}=18.2(8) \mathrm{s}$, and the ratio of the observed persistent flux to the net peak flux is $\gamma=F_{\text {pers }} / F_{\text {peak }}=0.021(1)$. The burst has the same spectral parameters as previous bursts observed for this source with HETE2 (Kawai \& Suzuki 2005) and RXTE (Galloway et al. 2005, 2006). Assuming a helium-burst at the Eddington limit (Lewin et al. 1992) and canonical NS parameters (1.4 solar mass and radius of $10 \mathrm{~km}$ ), we estimate the source distance to be $\sim 5 \mathrm{kpc}$.

\section{Discussion}

We analyzed the INTEGRAL and RXTE data of the accretionpowered millisecond pulsar HETE J1900.1-2455. We observed the source remaining in a bright state $\sim 136$ days after its discovery with a bolometric luminosity during our observation of 3-4 per cent of $L_{\text {Edd }}$ (Frank et al. 2002) (see Fig. 1 for the 
RXTE/ASM 1.5-12 keV light curve). The outburst light curve of HETE J1900.1-2455 is completely different from that of the other six observed MSPs which have shown exponential decays during the outburst: declining from peak luminosities of 3-30 per cent $L_{\text {Edd }}$ with a decay time-scale of several days up to $\sim 120$ days. Only HETE J1900.1-2455 has shown a fairly steady behavior at a low mass accretion rate (between 1 and 4 per cent of Eddington) with no sign of a transition toward quiescence. The most peculiar behavior of HETE J1900.1-2455 is the suppression of the pulsation at the NS spin period about a month from the beginning of the outburst (Kaaret et al. 2006); all other MSPs have had detectable pulsations throughout the duration of their outburst. The missing pulsations and the persistent X-ray emission at low mass accretion rate shows HETE J1900.1-2455 to be similar to the most commonly observed persistant, nonpulsating LMXB systems. It seems that the price to pay for being active over a long time is that pulsations are suppressed, i.e. there is an evident link between the long period of mass accretion and the missing pulsations.

The best spectral fit to the data required a two-component model, a cutoff PL or a thermal Comptonization model together with a soft component (see Table 1). The soft thermal emission detected at low energies could come either from the accretion disk or from a hot spot on the NS surface. As for the hard component, it most likely originates from the Comptonization of soft seed photons, $k T_{\text {seed }} \sim 1.5 \mathrm{keV}$ in a hot $k T_{\mathrm{e}} \sim 30 \mathrm{keV}$ plasma of moderate optical thickness $\tau_{\mathrm{T}} \sim 2$. The hard spectral component up to $200 \mathrm{keV}$ contributes most of the observed flux (70\%), even though a soft blackbody component is needed to fit the data. For the source distance of $5 \mathrm{kpc}$, the unabsorbed $0.1-300 \mathrm{keV}$ luminosity was (4.9-5.6) $\times 10^{36} \mathrm{erg} \mathrm{s}^{-1}$ (for different models).

We could not distinguish between the multi-temperature blackbody and single blackbody models, as both gave comparable parameters and $\chi^{2}$. However, for a distance of $5 \mathrm{kpc}$, our fit for a disk blackbody gives an inner disk radius, $R_{\text {in }} \sqrt{\cos i}=$ $2.6 \mathrm{~km}$, smaller than the expected NS radius. For blackbody emission, the fit implies an apparent area of the emission region $A_{\text {seed }} \sim 14 \mathrm{~km}^{2}$, which could be consistent with a heated NS surface around the accretion shock (Gierliński et al. 2002; Poutanen \& Gierliński 2003; Gierliński \& Poutanen 2005). On the other hand, the observed spectra before MJD 53558 are similar to those observed by us when pulsations are absent (Kaaret et al. 2006). They are also very similar to those of the atoll sources at low luminosities (Barret et al. 2000), where the X-rays are probably produced in the boundary/spreading layer near the NS equator (Kluźniak \& Wilson 1991; Inogamov \& Sunyaev 1999; Suleimanov \& Poutanen 2006).

Spectral similarities can be explained if in both types of sources (accreting MSPs and non-pulsating atoll sources), the energy dissipation happens in the optically thin medium (i.e. accretion shock and boundary/spreading layer) and the spectral properties are determined solely by energy balance and feedback from the NS surface which provides cooling in the form of soft photons (see e.g. Haardt \& Maraschi 1993; Stern et al. 1995; Poutanen \& Svensson 1996; Malzac et al. 2001). In these twophase models (cool neutron star with a hot dissipation region above), the product of electron temperature and optical depth is approximately constant. In HETE J1900.1-2455 we observe $k T_{\mathrm{e}} \times \tau_{\mathrm{T}} \approx 57 \mathrm{keV}$ (see Table 1) which is consistent with the values determined for other MSPs (Poutanen \& Gierliński 2003; Gierliński \& Poutanen 2005; Falanga et al. 2005a,b; Poutanen 2006) as well as with the theoretical models.

Since we found a fairly high blackbody temperature compared to other MSP sources, we attempted to fit a blackbody model with the soft emission being the source of the seed photons, i.e. $k T_{\mathrm{BB}}=k T_{\text {seed }}$. The obtained parameters are consistent with the other models, with similar optical depth, plasma temperature, and slightly lower soft thermal emission temperature. However, this fit implies a much larger apparent area of the seed photons, $A_{\text {seed }} \sim 240 \mathrm{~km}^{2}$, inconsistent with the whole NS surface.

Several X-ray bursts have been observed for this source by various observatories. These bursts are indicated with arrows in Fig. 1. The burst described in this work is similar in its properties to the other observed bursts as reported by Vanderspek et al. (2005); Barbier et al. (2005); Galloway et al. (2005, 2006). From the observed INTEGRAL burst properties and mass accretion rates inferred from the persistent luminosity, the present theory predicts that all these bursts are pure helium burning (e.g. Strohmayer \& Bildsten 2006). For helium flashes, the fuel burns rapidly, since there are no slow weak interactions, and the local Eddington limit is often exceeded. These conditions lead to PRE bursts with a duration, set mostly by the time it takes the heat to escape, of the order of 5-10 s, as observed. In the framework of the thermonuclear-flash models (e.g., Lewin et al. 1995) the burst duration, $\tau<20 \mathrm{~s}$, and the ratio of observed persistent flux to net peak flux $\gamma \approx 0.02$ indicate a hydrogen-poor burst.

Because there were no other bursts observed during the INTEGRAL observation, the burst recurrence time, $\Delta t_{\text {rec }}$, must be at least one day. We can compute the ratio of the total energy emitted in the persistent flux to that emitted in the burst

$\alpha=\frac{F_{\text {pers }}}{f_{\mathrm{b}}} \Delta t_{\mathrm{rec}}=\frac{\gamma}{\tau} \Delta t_{\mathrm{rec}}=\frac{0.021}{18.2} \Delta t_{\mathrm{rec}}>100$,

which is consistent with pure helium bursts (see e.g. Lewin et al. 1995). Taking the burst total energy release $E_{\mathrm{b}}=5 \times 10^{39} \mathrm{erg}$ (derived from the fluence $f_{\mathrm{b}}$ reported in Sect. 3.2.2) and He burning efficiency of $\epsilon_{\mathrm{He}} \approx 1.7 \mathrm{MeV} /$ nucleon $\approx 1.6 \times 10^{18} \mathrm{erg} \mathrm{g}^{-1}$, we can estimate the amount of fuel burned during the burst $E_{\mathrm{b}} / \epsilon_{\mathrm{He}} \sim 3.1 \times 10^{21} \mathrm{~g}$. For the mean mass accretion rate of 2 per cent of the Eddington through the entire outburst, a burst recurrence time of 2.2 days is expected. This is consistent with the observed frequency of the bursts (see Fig. 1 and note that not all bursts have been detected).

\section{Conclusions}

We have found that the source spectrum is similar to other accreting X-ray millisecond pulsars having a high plasma temperature around $30 \mathrm{keV}$ and a Thomson optical depth $\sim 2$. This source differs from other MSP in requiring thermal soft X-ray emission with nearly double the temperature. From our spectral fits we infer that this emission is not likely be produced in a multitemperature accretion disk but more likely arises from thermal emission at the NS surface. One might expect that the lack of pulsations could be due to a particularly high optical depth, but our spectral fits rule out this possibility.

The reason for the lack of coherent pulsations in the persistent emission from LMXBs is still an open question. Different explanations have been put forward to explain this phenomenon, including models which invoke gravitational lensing, electron scattering, or weak surface magnetic fields due to magnetic screening (Wood et al. 1988; Brainerd \& Lamb 1987; Titarchuk et al. 2000; Cumming et al. 2001, and references therein). The high accretion rate inferred for HETE J1900.1-2455 relative to the other known MSP transients suggests that we may be observing the evolution of the NS's magnetic field due to magnetic 
screening in this source (Cumming et al. 2001). The transition of HETE J1900.1-2455 from an X-ray millisecond pulsar to a persistent LMXB could indicate that there is a population of suppressed X-ray millisecond pulsars among the non-pulsating LMXBs. Detailed observations of this source at the epoch of pulsation suppression can help to solving the long-standing issue of missing pulsations in persistent LMXB emission.

Acknowledgements. M.F. acknowledges the French Space Agency (CNES) for financial support. J.P. acknowledges the Academy of Finland grants 102181 and 110792. E.W.B. is supported by Marie Curie Incoming European Fellowship contract MIF1-CT-2005-008762 within the 6th European Community Framework Programme.

\section{References}

Arnaud, K. A. 1996, in Astronomical Data Analysis Software and Systems V. ed. G. H. Jacoby, \& J. Barnes (San Francisco: ASP), ASP Conf. Ser., 101, 17 Barbier, L., Barthelmy, S., Cumming, J., et al. 2005, GCN Circ., 3824

Barret, D., Olive, J. F., Boirin, L., et al. 2000, ApJ, 533, 329

Brainerd, C. B., \& Lamb, F. K. 1987, 317, L33

Buccheri, R., Bennett, K., Bignami, G. F., et al. 1983, A\&A, 128, 245

Campana, S., Cucchiara, A., \& Burrows, D. 2005, ATel 535

Chakrabarty, D., Morgan, E. H., Muno, M. P., et al. 2003, Nature, 424, 42

Courvoisier, T. J.-L., Walter, R., Beckmann, V., et al. 2003, A\&A, 411, L57

Cumming, A., Zweibel, E., \& Bildsten, L. 2001, ApJ, 557, 958

Dickey, J. M., \& Lockman, F. J. 1990, ARA\&A, 28, 215

Falanga, M., Bonnet-Bidaud, J. M., Poutanen, J., et al. 2005a, A\&A, 436, 647

Falanga, M., Kuiper, L., Poutanen, J., et al. 2005b, A\&A, 444, 15

Frank, J., King, A., \& Raine, D. 2002, in Accretion Power in Astrophysics (Camb. Univ. Press)

Filippenko, A. V., Foley, R. J., \& Callanan, P. J. 2004, ATel 366

Fox, D. B. 2005, ATel 526

Galloway, D., Morgan, E., Kaaret, P., et al. 2005, ATel 657

Galloway, D., Muno, M. P., Hartman, J. M., et al. 2006, ApJS, submitted [arXiv: astro-ph/0608259]

Galloway, D., Morgan, E. H., Krauss, M. I., Kaaret, P., \& Chakrabarty, D. 2006, ApJ, in press [arXiv: astro-ph/0609693]

Gierliński, M., \& Poutanen, J. 2005, MNRAS, 359, 1261

Gierliński, M., Done, C., \& Barret, D. 2002, MNRAS, 331, 141

Goldwurm, A., David, P., Foschini, L., et al. 2003, A\&A, 411, L223
Gros, A., Goldwurm, A., Cadolle-Bel, M., et al. 2003, A\&A, 411, L179 Haardt, F., \& Maraschi, L. 1993, ApJ, 413, 507

Inogamov, N. A., \& Sunyaev, R. A. 1999, Astro. Lett., 25, 269

Jahoda, K., Swank, J. H., Giles, A. B., et al. 1996, Proc. SPIE, 2808, 59

Kaaret, P., Morgan, E. H., Vanderspek, R., \& Tomsick, J. A. 2006, ApJ, 638, 963

Kawai, N., \& Suzuki, M. 2005, ATel, 534

Kluźniak, W., \& Wilson, J. R. 1991, ApJ, 372, L87

Kuulkers, E., den Hartog, P. R., in 't Zand, J. J. M., et al. 2003, A\&A, 399, 663

Lebrun, F., Leray, J.-P., Lavocate, Ph., et al. 2003, A\&A, 411, L141

Levine, A. M., Bradt, H., \& Cui, W. 1996, ApJ, 469, L33

Lewin, W. H. G., van Paradijs, J., \& Taam, R. E. 1995, in X-ray Binaries, ed.

W. H. G. Lewin, J. van Paradijs, \& E. P. J. van den Heuvel (Cambridge: Cambridge Univ. Press), 175

Lund, N., Budtz-Joergensen, C., Westgaard, N. J., et al. 2003, A\&A, 411, L231

Malzac, J., Beloborodov, A. M., \& Poutanen, J. 2001, MNRAS, 326, 417

Mitsuda, K., Inoue, H., Koyama, K., et al. 1984, PASJ, 36, 741

Poutanen, J. 2006, Adv. Space Res., 38, 2697

Poutanen, J., \& Gierliński, M. 2003, MNRAS, 343, 1301

Poutanen, J., \& Svensson, R. 1996, ApJ, 470, 249

Roelofs, G., Jonker, P. G., Steeghs, D., Torres, M., \& Nelemans, G. 2004, ATel, 356

Rothschild, R. E., Blanco, P. R., Gruber, D. E., et al. 1998, ApJ, 496, 538

Rupen, M. P., Mioduszewski, A. J., \& Dhawan, V. 2005, ATel, 530

Suleimanov, V., \& Poutanen, J. 2006, MNRAS, 369, 2036

Steeghs, D., Torres, M. A. P., Garcia, M. R., et al. 2005a, ATel, 543

Steeghs, D., Torres, M. A. P., Blake, C., \& Bloom, J. S. 2005b, ATel, 530

Stern, B. E., Poutanen, J., Svensson, R., Sikora, M., \& Begelman, M. C. 1995, ApJ, 449, L13

Strohmayer, T. E., \& Markwardt, C. B. 1999, ApJ, 516, L81

Strohmayer, T. E., \& Bildsten, L. 2006, in Compact stellar X-ray sources, ed. W.

H. G. Lewin, \& M. van der Klis (Cambridge: Cambridge University Press), 113

Titarchuk, L., Cui, W., \& Wood, K. S. 1998, ApJ, 504, L27

Ubertini, P., Lebrun, F., Di Cocco, G., et al. 2003, A\&A, 411, L131

Vanderspek, R., Morgan, E., Crew, G., et al. 2005, ATel, 516

Verbunt, F., \& van den Heuvel, E. P. J. 1995, in X-ray binaries, ed. W. H. G.

Lewin, J. van Paradijs, \& E. P. J. van den Heuvel (Cambridge: Cambridge University Press), 457

Lewin, W. H. G. L., van Paradijs, J., \& Tamm, R. 1993, Space Sci. Rev., 62, 223 Wijnands, R. 2006, in Trends in Pulsar Research, ed. J. A. Lowry (NY: Nova Science Publishers), in press [arXiv:astro-ph/0501264]

Wijnands, R., \& van der Klis, M. 1998, Nature, 394, 344

Winkler, C., Courvoisier, T. J.-L., Di Cocco, G., et al. 2003, A\&A, 411, L1

Wood, K. S., Ftaclas, C., \& Kearney, M. 1988, ApJ, 324, L63 